\title{
New Sister Journal Kidney Cancer
}

The last few years have been notable for a marked upsurge of progress in genomics and molecular biology, producing tangible advances in understanding the underlying causes of urological cancers, leading in turn to new targets for potential future therapies. Reflecting this trend, we are pleased to announce the launch this month of a new sister journal to Bladder Cancer, Kidney Cancer (www.journalkidneycancer.com), dedicated to facilitating progress in understanding the epidemiology/etiology, genetics, molecular correlates, pathogenesis, pharmacology, ethics, patient advocacy and survivorship, diagnosis and treatment of tumors of the kidney. The journal will be available free in 2017 so you will be able to see the early issues of the journal for yourself.

Kidney Cancer has two excellent Editors-inChief in Primo Lara and Peter Mulders, as well as the support of an outstanding Editorial Board (see www.journalkidneycancer.com/editorialboard). The launch issue features an impressive array of research articles and reviews, along with Clinical Trials Corner (www.journalkidneycancer. com/clinical-trials) featuring news from the most interesting clinical trials, an idea borrowed from Bladder Cancer. Like Bladder Cancer, the sister journal also features short communications, and letters to the editor. Also like Bladder Cancer, it offers rigorous peer review and rapid online pre-print publication on acceptance.
We are delighted by the addition of this sister journal to the IOS family of cancer journals, especially in view of the high quality of the new arrival. As with Bladder Cancer, we feel confident that Kidney Cancer will be an influential force for good in translating advances in our understanding into new and more effective treatments for urological cancers in the coming years.

Editors-in-Chief

Seth P. Lerner Scott Department of Urology Baylor College of Medicine Houston, Texas, USA E-mail: blc@iospress.com.

Dan Theodorescu University of Colorado Cancer Center Aurora, Colorado, USA E-mail: blc@iospress.com.

\section{Submission of Papers}

Prospective authors for Kidney Cancer are requested to submit their manuscript electronically the online submission system msTracker: http://msTracker.com/submit1.php?jc=kca and for detailed instructions to authors, visit (www.journal kidneycancer.com/submission-guidelines). 